\title{
Ethical dilemmas associated with the introduction of biologic treatments in chronic rhinosinusitis with nasal polyps*
}

Claire Hopkins

ENT Department, Guy's Hospital, London, United Kingdom
Rhinology 60: 3, $162-168,2022$

https://doi.org/10.4193/Rhin21.477

*Received for publication:

December 23, 2021

Accepted: January 16, 2022

\begin{abstract}
This review considers ethical dilemmas which may present when introducing the use of biologic treatments alongside existing treatments in the management of chronic rhinosinusitis with nasal polyps. Biologic therapies offer hope to patients with diseases recalcitrant to conventional therapies but are often significantly more expensive. Reducing the need for surgery may act as a disincentive for use within surgical specialities, while reimbursement for administration has the potential to encourage inappropriate use.

Any treatment should be used considering the principles of beneficence (offering the most effective treatment to the patient) and non-maleficence (minimising the risk of harm); the challenges of comparing the different available treatment options are considered.

Patient autonomy should be involved a process of shared decision making, but when a third-party payor is involved they may seek to place restrictions on access to treatments that limit the choice of both patient and physician. Such decisions are often based on the cost-effectiveness of novel treatments relative to standard of care; published models suggest that at current market prices, biologics are less cost-effective in all groups that standard care. Social justice (fair distribution of limited healthcare resources) therefore may mandate rationing of access.

To this end, working as part of professional organisations or research groups, physicians often produce guidelines that help to identify those in greatest need of novel treatments. The challenges in creating and applying these guidelines are also considered.
\end{abstract}

Key words: biologics, chronic rhinosinusitis, ethics, guidelines, treatment

\section{Introduction}

The introduction of novel treatments provides an opportunity to treat those in whom there were previous no effective therapies, or to improve outcomes or reduce the risk of adverse events associated with pre-existing treatments. However, at the same time there is a risk that indications for treatment may be extended such that treatment is offered to those who are unable to derive significant benefit, that novel treatments may be offered by healthcare practitioners less familiar with the disease or who are unable to offer all therapeutic options. There are also risks that adoption of novel procedures may be financially motivated; a recent systematic review has shown a clear dose response relationship between prescribing and financial payments from the pharmaceutical industry(1) and there is evidence that industry relationships are associated with the adoption of use of balloon sinuplasty ${ }^{(2)}$. Conversely, loss of revenue may discourage use of non-surgical interventions that may reduce the requirement for surgery, particularly for conditions such as chronic rhinosinusitis with nasal polyps (CRSwNP) that are associated with a high revision rate ${ }^{(3)}$. It is therefore important to consider where such dilemmas may arise in the introduction of biologics and other novel treatments in the management of CRSwNP when developing guidelines to aid decision making.

The need to benefit patients (beneficence) while avoiding harm (non-maleficence), respecting patient choice (autonomy) and achieving fair, equitable treatment of limited health-care resources (justice) are the four principles that underpin ethical 


\section{Beneficence}

Choosing therapy in the best interests of the patient

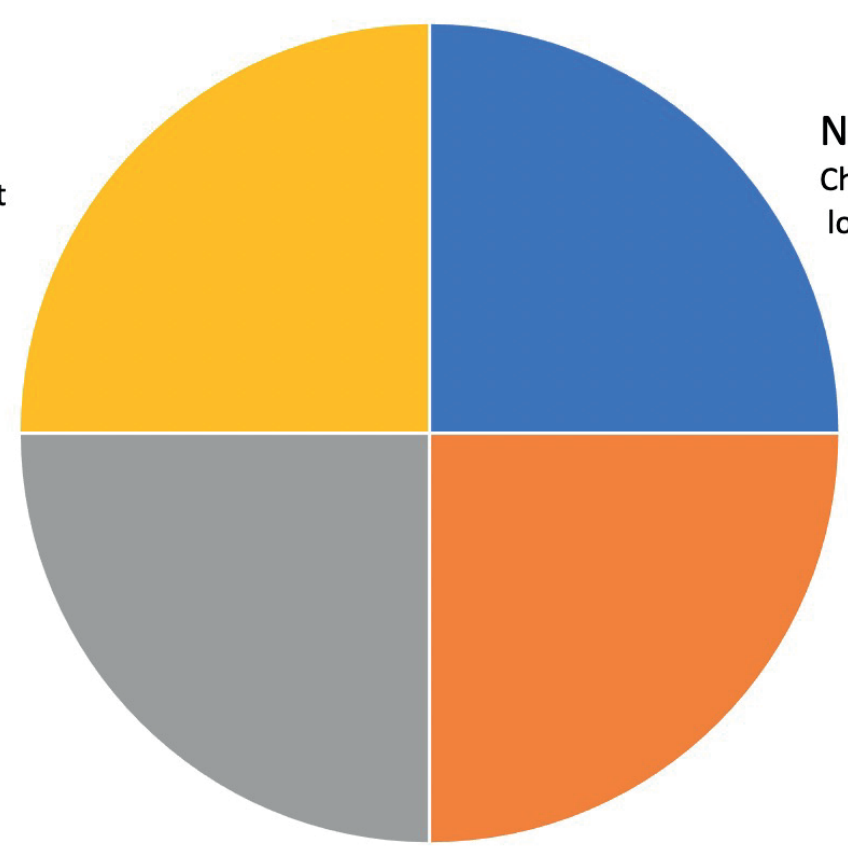

\section{Non-maleficence Choosing therapy with the lowest risk of harm}

\section{Autonomy} Patient preference

\author{
Justice \\ Ensuring fair distribution of \\ scarce health resources
}

Figure 1. The four pillars of ethical decision making in clinical practice.

decision making in clinical practice ${ }^{(4)}$ (Figure 1).

\section{Beneficence and non-maleficence}

Choosing treatment in the best interests of the patient must always balance the risk of adverse events. Intranasal corticosteroids have a low risk of harm but limited effect on rhinorrhoea or loss of smell ${ }^{(5)}$. Oral steroids often achieve significant reduction in symptoms of nasal polyps, but the benefit is often shortlived $^{(6)}$ and there is a growing awareness of the risks of harms $s^{(7)}$ which accumulate with repeated use ${ }^{(8)}$, with a little as four courses over a life-time associated with increased risk of harm. Sinus surgery may achieve large reductions in symptom scores but carries a risk of major complications in 1 in 250 patients $^{(9)}$, and revision surgery rates more than $20 \%$ at 5 years. Nonetheless, sinus surgery may have a disease modifying effect in that it improves the effectiveness of intranasal corticosteroids ${ }^{(10)}$ and nearly $80 \%$ of patients have not undergone revision in the first five years after surgery, although many may not be adequately controlled $^{(11)}$. There has been a paucity of comparative studies of medical versus endoscopic surgical treatment of CRSwNP, however a recent trial highlights that both strategies are associated with high levels of uncontrolled disease ${ }^{(12)}$.

To this end, there has been growing interest in the use of monoclonal antibodies that target type 2 inflammation in the search to achieve better disease control in severe CRSwNP(13-16), and have been shown to reduce nasal polyp scores and nasal obstruction.

Three biologics have currently been approved for use by the
Federal Drug Administration (US) and European Medicines Agency. Certainly, the risk of adverse events appear low, results appear to maintained for the duration of treatment and the need for surgery and oral corticosteroids is significantly redu$\operatorname{ced}^{(17,18)}$. However, treatment must be continued, potentially life long, to sustain improvements as there is currently no evidence of a disease modifying effect and not all patients respond. It is not possible to predict responders prior to a trial of treatment. One real-life registry of patients treated with biologics for their asthma who had co-existing CRSwNP found that more than 20\% stilled require surgical intervention ${ }^{(19)}$; most patients received omalizumab or mepolizumb. In contrast, a more recent registry of CRSwNP patients treated with dupilumab ${ }^{(20)}$ reported very low rates of rescue OCS usage, surgery or poor-response.

It is difficult to compare the outcomes of trials of different biologics, either with each other or other treatment strategies. Inclusion criteria differ leading to widely different baseline cohorts, leading to different potential floor and ceiling effects. Different grading systems for nasal polyp score have been utilised, there are different restrictions both on use of rescue medication or surgery and statistical methods to deal with this (such as using the worst observation for patients receiving rescue oral steroids). Some outcomes, such as psychophysical olfactory testing using UPSIT tests, are more easily compared between trials; improvements of 7.9 points are reported in a meta-analysis of outcomes of sinus surgery for CRSwNP(21), while greater improvements, 11.3 points, were reported using dupilumab. However, the baseline severity of patients was more severe in the dupilumab study 


\section{Satisfaction with currently available treatment options}

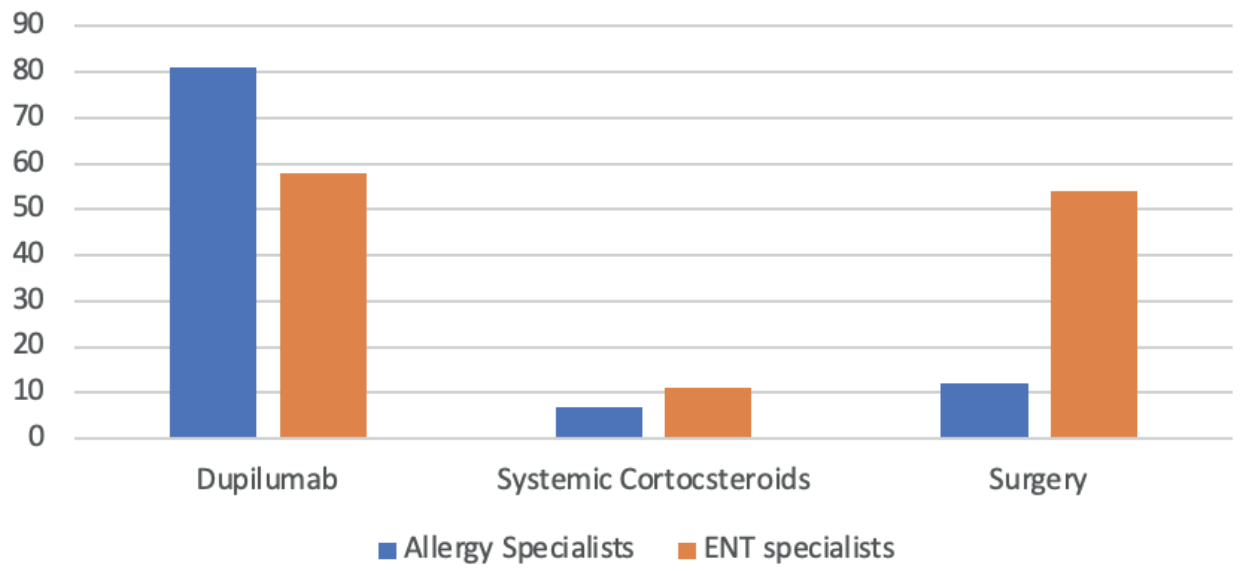

Figure 2. Results of unpublished market research made available by personal communication and used with permission from Sanofi. Results displayed as number of physicians rating satisfaction at 6 or 7 on a 7-point Likert scale.

with $74 \%$ considered anosmia at baseline. When only anosmic patients are considered, surgery achieved an 11.8-point improvement In UPSIT scores. This example highlights the challenges in interpreting the current evidence base to inform our patients of relative risks and benefits of the treatment options available.

\section{What may influence our interpretation of the evi- dence base?}

An unpublished market research study (personal communication, Sanofi) surveyed US-based allergists and ENT surgeons regarding their satisfaction with current treatment options for CRSwNP. Perhaps unsurprisingly, there were low rates of satisfaction with systemic corticosteroids across both groups (Figure 2). However, the responses for dupilumab and surgery clearly differed between groups, with allergists reporting much higher levels of satisfaction with dupilumab than surgery while ENT specialists rated both similarly, albeit with less enthusiasm for Dupilumab than their colleagues. When questioned further on the response to surgery ENT surgeons felt that $33 \%$ of patients had more than 3 years symptomatic relief after surgery with only $11 \%$ failing to benefit, while allergists rated only $18 \%$ of patients as receiving durable benefit while $24 \%$ did not respond.

Why the differences? Could it be that views are unduly influenced by financial rewards and a desire to keep the patient within their own practices? More likely, it simply reflects observation bias; if a patient receives significant benefit from surgery, maintained over several years, they will likely return to the same ENT surgeon should their symptoms recur many years later. In contrast, if they failed to achieve meaningful improvement or recur soon after surgery thy will be more likely to seek an alternative approach with an allergist. This will likely influence prescribing preferences; indeed patients describe feeling "like the rope in a tug or war game; my ENT and my allergist are pushing me in different directions regarding treatment ${ }^{\prime \prime 22)}$. Patient reports detail how ENT surgeons may be reluctant to consider biologics, citing lack of evidence of effectiveness, or concerns of yet unknown side effects. In contrast allergists are reportedly prescribing even without seeing the polyps, or in paucisymptomtic patients. Patients go as far as to question whether there is a financial motivation behind the very different attitudes. It is clear that all specialty groups should work together, and patients are best managed within a multidisciplinary framework to ensure that all different treatment options are discussed in a balanced manner in order to allow the patient to make an informed choice. Furthermore, guidelines should include all stakeholders and should be free from commercial bias.

\section{Autonomy}

Shared decision-making dictates that the patient's values and preferences to be considered when choosing between different treatment options, although policy may determine which options are available for them to choose from. Qualitative studies show a wide range of preferences, with some patients keen to avoid an 'endless surgery merry-go-round', while others are more fearful of the risks of taking oral steroids or biologic 
therapies, particularly over long periods ${ }^{(22,23)}$.

Interestingly, a consensus guideline published earlier in the year specifically acknowledges patient autonomy and creates a pathway for patients whose symptoms are refractory to intranasal steroids to decline surgical intervention and start treatment with a biologic ${ }^{(24)}$.

Whether third-party payors support such decisions will vary from country to country as the need for distributive justice may over-ride patient autonomy in some settings.

\section{Justice}

In a system with a finite amount of healthcare resources. there is a need to ensure fair and equitable distribution, usually based on clinical needs. This is particularly relevant when considering the high costs of long-term use of biologic agents, relative to the cost of sinus surgery and intranasal corticosteroids. Biologics alone accounted for nearly $40 \%$ percent of U.S. prescription drug spending in $2015^{(25)}$, but were prescribed to less than $2 \%$ of the population and for 70 percent of drug spending growth between 2010 and 2015(26).

There is evidence post-marketing drug prices have increased significantly for many biologics since FDA approval and even pre-existing conventional disease modifying drug prices have risen exponentially in parallel with the introduction of biologics to 'keep up'(27). With an aging population and growing number of chronic conditions that are amenable to biological therapies, achieving distributive justice presents a significant challenge.

In the absence of comparative studies comparing biologic therapy to a surgical strategy health-economic models may attempt to create estimates of cost-effectiveness. Such models assume a certain rate of complications with each intervention and build in a recurring rate of revision surgery. One analysis estimated that the surgical strategy achieved 9.8 quality adjusted life years (QALYs) at a cost of $\$ 50,437$ while dupilumab produced 8.95 QALYs for $\$ 536,420$; surgery remains the more cost-effective choice for any yearly cost of a biologic greater than $\$ 855(28)$. Even when considering patients known to be at the highest risk of recurrence, such as those with NSAID exacerbated respiratory disease, surgery and desensitisation was significantly more cost-effective then either up-front or salvage use of dupilumab(29).

There are limitations to such models. They likely underestimate the true cost of a surgical strategy as they exclude indirect costs associated with absenteeism due to post-operative recovery and fails to account for the heterogeneity in surgery offered which may lead to differing revision rates. There are hidden costs from adverse events related to repeated use of oral corticosteroids; in an Italian study these costs were estimated to total nearly EU250 million per annum for severe asthmatics. They also neglect the added benefit on comorbid type 2 conditions such as asthma. Similarly, they assume a $100 \%$ response rate to biologics and avoidance of the need for surgery, which is not supported by the current literature ${ }^{(13-16,19)}$. They do not account for steroid-eluting stents or other interventions that may reduce need for primary or revision surgery. A further limitation is that many metrics used to calculate health utility, such as the EQ-5D, likely fail to capture the quality-of-life impact of loss of smell, both directly and indirectly through its associated adverse impact on mental health ${ }^{(30)}$.

However, there is a clear need to improve cost-effectiveness if wider market access is desirable aim. Research is required to determine whether dosing intervals can be extended de novo or once control of the disease has been achieved. A study in atopic dermatitis, another type 2 disease, found a dose-dependent reduction in response rate in those on 4 and 8 weekly regimens compared to a 2-weekly interval ${ }^{(31)}$. In contrast, the results of the 52-week phase 3 study of dupilumab used for severe CRSwNP suggests that benefits were maintained in a cohort who were moved onto a 4-weekly regimen after 24 weeks of 2 weekly treatments $^{(16)}$. A real-life registry of CRSwNP patients treated with dupilumab reported that over $90 \%$ patients could be moved to a 4 weekly regimen after 36 weeks of treatment, and $46 \%$ could be maintained by treatments given at 6 weekly intervals after 48 weeks $^{(20)}$. Hopes that biosimilars may reduce costs once the originator biologic patent has expired have not been realised in the rheumatological market space - biosimilars still account for less than $1 \%$ of anti-TNF sales in the US ${ }^{(32)}$. This likely occurs due to a combination of ongoing attempts to block entry to market by pharmaceutical companies, use of rebate systems, non-competitive pricing of the biosimilars and reluctance on the part of physicians to change prescribing habits (physicians' reimbursement for administering drugs in office are often based on a percentage of price. Single market payor systems have achieved greater success by mandating switching to biosimilars once available.

Alternative strategies are therefore required to achieve distributive justice. One method is to identify and target those patients who are least likely to benefit from conventional treatment strategies. N-ERD is associated with significantly higher risk of revision surgery ${ }^{(33)}$, research suggests that patients with N-ERD achieve greater improvements in nasal congestion, total symptom scores, radiological severity and SNOT-22 scores than those without. Patients with a history of previous surgery, particularly those that have a symptomatic recurrence that requires revision within 3 years, are at a higher risk of undergoing further surgical intervention ${ }^{(33)}$; again a recent study has shown that while all groups benefit from dupilumab, those with a history of increasing numbers of previous surgery, or reducing time since last surgery to revision benefit the most ${ }^{(34)}$. It appears that characte- 
ristics that predict failure of conventional treatment strategies or early recurrence may also help to identify those who derive greater benefit from biological therapies, although further work to predict responders is required.

Head-to-head studies and real-world registries are required to accurately evaluate the relative costs of each treatment pathway. It will be important not to focus only on biologics versus surgery, as there may be merit in combining both - for example reducing the inflammatory load with surgery might allow for biologics to maintain benefit and prevent recurrence with a longer interval between doses. However, 'cost-effectiveness' may be unattainable, and therefore payors may seek to restrict access to those refractory to or ineligible for more conventional treatment strategies.

\section{Who should decide who receives biologic therapy for CRSwNP?}

Intrinsic to the doctor-patient relationship is the duty of the physician to prioritise the interests of the patient in front of them. This may be enshrined in law, for example in Canada, in the face of budgetary restrictions "if it comes to a choice between a physician's responsibility to his or her individual patient and his or her responsibility to the medicare system overall, the former must take precedence ${ }^{\prime \prime(35)}$. However, it is essential that physicians inform the development of any policies applied by payors that seek to restrict access, lest they be driven purely by cost-constraint or a desire to mitigate against the risk of legal challenge. To this aim, and number of treatment algorithms have been proposed by different expert panels $(24,36-38)$.

In creating such guidance, it is important to consider the same ethical domains discussed above:

- Identifying all patients who will likely benefit from a biologic while excluding those who will not respond

- Identifying patients who derive least benefit, or are at risk of harm from alternative strategies

- Allowing patient preference to contribute to decision making

- Considering the costs of the treatment relative to the healthcare resources available.

Most guidelines share an attempt to identify patients with CRSwNP with Type 2 inflammation, although this is limited by the absence of specific cut-offs for biomarkers such as serum eosinophil count. They attempt to minimise harm by incorporating the number of courses of oral steroids and targeting the use of biologics to those who require repeated courses. They generaIly recommend use only in those with more severe symptoms, defining a threshold SNOT-22 or VAS score in some cases.
They differ slightly in the positioning of biologics relative to surgery; EPOS 2020 $0^{(38)}$ largely restricts use of biologics to those that have failed conventional surgery, a US based consensus allows for the right for patients to decline surgery(24) and the most recent EUFOREA guideline considers all patients with severe CRSwNP regardless of previous surgery. There is no contraindication in starting a biologic before surgery has been undertaken, and the position relative to surgery largely depends on the resources available. In countries with more limited resources, additional limitation may be recommended to ensure that the most severely affected patients are able to access treatment(39). As costs change and evidence accrues, guidelines will need to be updated.

\section{Ethical dilemmas in applying treatment guidelines in clinical practice}

There is emerging evidence that more extensive surgery may be associated with lower long term revision surgery rates ${ }^{(33,40)}$. To this end, some guidelines ${ }^{(24)}$ include caveats regarding the type of surgery that should have been undertaken before considering biologic therapy. The US Multidisciplinary Consensus includes a statement 'if symptoms persist despite appropriate sinus surgery, consider....", and in the discussion suggests that the completeness of prior surgery is a factor to be considered in the decision making process. However, 'appropriate surgery' is not defined, no guidance is given regarding who should assess if previous surgery has been adequate nor what to do if deemed to be inadequate. This could create a situation where surgeons are asked to effectively mark their own homework, or to judge the work of colleagues. In the worst case, it might incentivise unethical surgeons to perform only limited surgery, likely to be associated with higher revision rates, but potentially also allowing them to exclude the patient from biologic therapies. It is hard to achieve the aim of patient autonomy when insisting that a patient undergoes revision surgery when the first has failed, because, through no fault of their own, their first surgery was deemed to be inadequate. Careful thought will be required before this can be applied in clinical practice. In the interim it is important that we train our future generations of surgeons to undertake 'complete' endoscopic sinus surgery where indicated and to recognise which patients might benefit from treatment within specialist centres.

If biologics are to be prescribed, the role of planned surgery before or shortly after initiation of treatment remains unclear. As above, there may be a role for combining surgery and biological therapy with a view to being able to reduce frequency of treatment, however there is no evidence to support this approach yet. As response to biologics is often rapid, with improvements often seen within the first 8 weeks in many of those who respond $^{(16)}$, and given that one of the aims of biologic treatment 
is to reduce the need for both surgery and oral corticosteroids, it seems very reasonable to allow a period of observation after commencing treatment, and reserving surgery only for those who fail to respond, or have a partial respond with residual polyps, as recommended in the EUFOREA 2021 guidelines(36). Despite this, there are anecdotal reports of ENT surgeons insisting that surgery is essential despite significant improvements within weeks of starting biological therapy. Given that ongoing treatment with a biologic can achieve significant reductions in polyp size and radiological extent of disease, this approach currently seems difficult to justify.

\section{Where do we go from here?}

As health-care providers we have a duty to adopt novel treatments in keeping with the ethical pillars described above. As individuals we must advocate for our patients, and to so we must understand the risks and benefits of all available treatment options and discuss these with our patients in a balanced manner, free from bias, and preferably in a multidisciplinary setting. Specialty associations must guide members and payors through developing evidence-based guidelines. Researchers must help build the strong evidence base which underpins them., and work with pharmaceutical companies to help ensure that future trials address issues relevant to the patient, and include outcomes that facilitate comparison between trials. The pharmaceutical companies must ensure fair pricing and resist from blocking the entry of biosimilars into the marketplace when patents expire.

There is no doubt that biologic treatments are valuable tools in the management of CRSwNP. With careful consideration we can help to ensure that their potential benefits are directed at those who will derive greatest benefit.

\section{Acknowledgements}

None.

\section{Authorship contribution}

This review is based on the lecture presented by $\mathrm{CH}$ as one of the plenary lectures at the ERS-ISIAN 2021 Congress in Thessaloniki, Greece

\section{Conflict of interest}

No conflicts of interests exists.

\section{Funding resources}

None.

\section{References}

1. Mitchell AP, Trivedi NU, Gennarelli RL, Chimonas S, Tabatabai SM, Goldberg $J$, et al. Are Financial Payments From the Pharmaceutical Industry Associated With Physician Prescribing? : A Systematic Review. Ann Int Med. 2021;174(3):353-61.

2. Eloy JA, Svider PF, Bobian M, Harvey RJ, Gray ST, Baredes S, et al. Industry relationships are associated with performing a greater number of sinus balloon dilation procedures. Int Forum Allergy Rhinol. 2017;7(9):878-83.

3. Hopkins C, Slack R, Lund V, Brown P, Copley $L$, Browne J. Long-term outcomes from the English national comparative audit of surgery for nasal polyposis and chronic rhinosinusitis. Laryngoscope. 2009;119(12):245965.

4. Varkey B. Principles of Clinical Ethics and Their Application to Practice. Medical principles and practice. Int J Kuwait University, Health Science Centre. 2021;30(1):17-28.

5. Chong LY, Head K, Hopkins C, Philpott C, Schilder AG, Burton MJ. Intranasal steroids versus placebo or no intervention for chronic rhinosinusitis. Cochrane Database Syst Rev. 2016:4:CD011996.

6. Head K, Chong LY, Hopkins C, Philpott C, Burton MJ, Schilder AG. Short-course oral steroids alone for chronic rhinosinusitis. Cochrane Database Syst Rev. 2016:4:CD011991.

7. Hox V, Lourijsen E, Jordens A, Aasbjerg K,
Agache I, Alobid I, et al. Benefits and harm of systemic steroids for short- and longterm use in rhinitis and rhinosinusitis: an EAACI position paper. Clin Transl Allergy. 2020;10:1.

8. Price D, Castro M, Bourdin A, Fucile $S$ Altman P. Short-course systemic corticosteroids in asthma: striking the balance between efficacy and safety. Eur Resp Rev. 2020;29(155)

9. Hopkins C, Browne JP, Slack R, Lund VJ, Topham J, Reeves BC, et al. Complications of surgery for nasal polyposis and chronic rhinosinusitis: the results of a national audit in England and Wales. Laryngoscope. 2006;116(8):1494-9.

10. Snidvongs $K$, Kalish L, Sacks R, Sivasubramaniam R, Cope D, Harvey RJ. Sinus surgery and delivery method influence the effectiveness of topical corticosteroids for chronic rhinosinusitis: systematic review and meta-analysis. Am J Rhinol Allergy. 2013;27(3):221-33.

11. van der Veen J, Seys SF, Timmermans $M$, Levie P, Jorissen M, Fokkens WJ, et al. Reallife study showing uncontrolled rhinosinusitis after sinus surgery in a tertiary referral centre. Allergy. 2017;72(2):282-90.

12. Lourijsen E, Reitsma S, Vleming $M$, et al Endoscopic Sinus Surgery with medical therapy versus medical therapy for chronic rhinosinusitos with nasal polyps: a multicentre, randomised, comtrolled trial. Lancet Respir Med. 2022:S2213-2600(21)00457-4
13. Han JK, Bachert C, Fokkens W, Desrosiers M, Wagenmann M, Lee SE, et al. Mepolizumab for chronic rhinosinusitis with nasal polyps (SYNAPSE): a randomised, double-blind, placebo-controlled, phase 3 trial. Lancet Respir Med. 2021;9(10):1141-53.

14. Gevaert P, Omachi TA, Corren J, Mullol J, Han J, Lee SE, et al. Efficacy and safety of omalizumab in nasal polyposis: 2 randomized phase 3 trials. J Allergy Clin Immunol. 2020;146(3):595-605.

15. Bachert C, Han JK, Desrosiers MY, Gevaert $P_{\text {, }}$ Heffler E, Hopkins C, et al. Efficacy and safety of benralizumab in chronic rhinosinusitis with nasal polyps: A randomized, placebo-controlled trial. J Allergy Clin Immunol. 2021;S0091-6749(21)01459-7.

16. Bachert C, Hellings PW, Mullol J, Naclerio RM, Chao J, Amin N, et al. Dupilumab improves patient-reported outcomes in patients with chronic rhinosinusitis with nasal polyps and comorbid asthma. J Allergy Clin Immunol Practice. 2019;7(7):2447-9 e2.

17. Chong LY, Piromchai P, Sharp S, Snidvongs K, Webster KE, Philpott C, et al. Biologics for chronic rhinosinusitis. Cochrane Database Syst Rev. 2021;3:CD013513.

18. Desrosiers M, Mannent LP, Amin $N$, Canonica GW, Hellings PW, Gevaert P, et al. Dupilumab reduces systemic corticosteroid use and sinonasal surgery rate in CRSwNP. Rhinology. 2021:59(3):301-11.

19. Bajpai S, Marino MJ, Rank MA, Donaldson 
AM, O'Brien EK, Lal D. Benefits of biologic therapy administered for asthma on Coexistent chronic rhinosinusitis: A realworld study. Int Forum Allergy Rhinol. 2021;11(8):1152-61.

20. Lans R, Fokkens WJ, Adriaensen G, Hoven DR, Drubbel JJ, Reitsma S. Real-life observational cohort verifies high efficacy of dupilumab for Chronic Rhinosinusitis with Nasal Polyps. Allergy. 2021;77(2):670-674.

21. Kohli P, Naik AN, Farhood Z, Ong AA Nguyen SA, Soler ZM, et al. Olfactory Outcomes after Endoscopic Sinus Surgery for Chronic Rhinosinusitis: A Metaanalysis. Otolaryngol Head Neck Surg. 2016;155(6):936-48

22. Alanin MC, Laidlaw T, Society TS, Hopkins C The Burden of Non-steroidal anti-inflammatory exacerbated respiratory disease from the patient's perspective - a qualitative analysis of posts from the Samter's Society. Rhinology. 2020;58(4):333-40.

23. Vennik J, Eyles C, Thomas M, Hopkins C Little P, Blackshaw $\mathrm{H}$, et al. Chronic rhinosinusitis: a qualitative study of patient views and experiences of current management in primary and secondary care. BMJ Open. 2019;9(4):e022644.

24. Han JK, Bosso JV, Cho SH, Franzese C, Lam K, Lane AP, et al. Multidisciplinary consensus on a stepwise treatment algorithm for management of chronic rhinosinusitis with nasal polyps. Int Forum Allergy Rhinol. 2021;11(10):1407-16.

25. Allan Coukell SDaHCC, "Pew Charitable Trusts, Drug Spending Research Initiative, November 16, 2015. As of September 27, 2017: http://www.pewtrusts.org/en/ research-and-analysis/fact-sheets/2015/11/ specialty-drugs-and-health-care-costs.

26. National Pharmaceutical Services SDS, " white paper, undated. As of September 27 2017: https://www.pti-nps.com/nps/wpcontent/uploads/2017/04/NPS_Specialty-
Medication-White-Paper-Web.pdf.

27. Dalal DS, Zhang T, Shireman TI. Medicare expenditures for conventional and biologic disease modifying agents commonly used for treatment of rheumatoid arthritis. Semin Arthritis Rheum. 2020:50(5):822-6.

28. Scangas GA, Wu AW, Ting JY, Metson R, Walgama E, Shrime MG, et al. Cost Utility Analysis of Dupilumab Versus Endoscopic Sinus Surgery for Chronic Rhinosinusitis With Nasal Polyps. Laryngoscope. 2021;131(1):E26-E33.

29. Yong M, Wu YQ, Howlett J, Ballreich J, Walgama E, Thamboo A. Cost-effectiveness analysis comparing dupilumab and aspirin desensitization therapy for chronic rhinosinusitis with nasal polyposis in aspirinexacerbated respiratory disease. Int Forum Allergy Rhinol. 2021;11(12):1626-36.

30. Chung JH, Lee YJ, Kang TW, Kim KR, Jang DP, Kim IY, et al. Altered Quality of Life and Psychological Health (SCL-90-R) in Patients With Chronic Rhinosinusitis With Nasal Polyps. Ann Otol Rhinol Laryngol. 2015;124(8):663-70.

31. Worm M, Simpson EL, Thaci D, Bissonnette R, Lacour JP, Beissert S, et al. Efficacy and Safety of Multiple Dupilumab Dose Regimens After Initial Successful Treatment in Patients With Atopic Dermatitis: A Randomized Clinical Trial. JAMA Dermatol. 2020;156(2):131-43.

32. Yazdany J. Failure to Launch: Biosimilar Sales Continue to Fall Flat in the United States. Arthritis Rheumatol. 2020;72(6):870-3.

33. Hopkins C, Lund V. Does time from previous surgery predict subsequent treatment failure in Chronic Rhinosinusitis with Nasal Polyps? Rhinology. 2021;59(3):277-83.

34. Hopkins C, Wagenmann M, Bachert C, Desrosiers M, Han JK, Hellings PW, et al. Efficacy of dupilumab in patients with a history of prior sinus surgery for chronic rhinosinusitis with nasal polyps. Int Forum
Allergy Rhinol. 2021;11(7):1087-101.

35. Law Estate v. Simice (1994) CCLTdBCSC, aff'd [1996] 4 W.W.R. 672 (C.A.).

36. Bachert C, Han JK, Wagenmann M Hosemann W, Lee SE, Backer V, et al. EUFOREA expert board meeting on uncontrolled severe chronic rhinosinusitis with nasal polyps (CRSWNP) and biologics: Definitions and management. J Allergy Clin Immunol. 2021;147(1):29-36.

37. Fokkens WJ, Lund V, Bachert C, Mullol J, Bjermer L, Bousquet J, et al. EUFOREA consensus on biologics for CRSWNP with or without asthma. Allergy. 2019;74(12):2312-9.

38. Fokkens WJ, Lund VJ, Hopkins C, Hellings PW, Kern R, Reitsma S, et al. European Position Paper on Rhinosinusitis and Nasal Polyps 2020. Rhinology. 2020;58(Suppl S29):1-464.

39. Hopkins C, McKenzie JL, Anari S, Carrie $S$, Ramakrishnan $Y$, Kara $N$, et al. British Rhinological Society Consensus Guidance on the use of biological therapies for chronic rhinosinusitis with nasal polyps. Clin Otolaryngol. 2021;46(5):1037-43.

40. Zhang L, Zhang Y, Gao Y, Wang K, Lou H, Meng $Y$, et al. Long-term outcomes of different endoscopic sinus surgery in recurrent chronic rhinosinusitis with nasal polyps and asthma. Rhinology. 2020;58(2):126-35.

Prof Claire Hopkins

ENT Department

Guy's Hospital

London

SE1 9RT

United Kingdom

E-mail: clairehopkins@yahoo.com 